\title{
Chronic lipopolysaccharide exposure induces cognitive dysfunction without affecting BDNF expression in the rat hippocampus
}

\author{
BIN ZHU ${ }^{1}$, ZHI-GANG WANG ${ }^{2}$, JIE DING $^{3}$, NING LIU ${ }^{1}$, DA-MING WANG ${ }^{1}$, \\ LIANG-CAI DING ${ }^{1}$ and CHUN YANG ${ }^{3}$ \\ Departments of ${ }^{1}$ Critical Care Medicine, ${ }^{2}$ Respiratory Medicine and ${ }^{3}$ Anesthesiology, \\ The Third Affiliated Hospital of Soochow University, Changzhou, Jiangsu 213003, P.R. China
}

Received August 13, 2013; Accepted January 2, 2014

DOI: $10.3892 /$ etm.2014.1479

\begin{abstract}
Previous studies have shown that lipopolysaccharide (LPS) has the potential to cause cognitive dysfunction. However, the underlying pathogenesis has yet to be fully elucidated. Increasing attention is being focused on infection in the central nervous system. Therefore, the present study aimed to investigate the behavioral performance of rats receiving intraperitoneal injections of LPS and to determine the expression levels of amyloid- $\beta(\mathrm{A} \beta)$, brain-derived neurotrophic factor (BDNF) and pro-inflammatory cytokines in the hippocampus. In total, 30 male Wistar rats were randomly divided into 3 groups (each $n=10$ ): Control and 3 and 7 day LPS administration groups. The rats were intraperitoneally injected with saline or LPS for 3 or 7 days. Following this, rats performed the Morris water maze test, in which the latency to the platform and proportion of time spent in the target quadrant were recorded. Rats were then sacrificed and the hippocampi were harvested for determination of interleukin (IL)-1 $\beta$, IL- 6 , tumor necrosis factor- $\alpha$ (TNF- $\alpha$ ), A $\beta$ and BDNF expression levels. LPS administration for 3 and 7 days significantly increased the latency to the platform and decreased the proportion of time spent in the target quadrant compared with those in the control group, $(\mathrm{P}<0.05)$. Administration of LPS for 3 and 7 days induced statistically significant increases in the expression levels of IL-1 $\beta$, IL- 6 and TNF- $\alpha$ in the hippocampus, compared with those in the control group $(\mathrm{P}<0.05)$. Additionally, the administration of LPS for 7 days induced a statistically significant increase in the expression level of $A \beta$ in the hippocampus, compared with that in the control group
\end{abstract}

Correspondence to: Dr Chun Yang, Department of Anesthesiology, The Third Affiliated Hospital of Soochow University, 185 Juqian Street, Changzhou, Jiangsu 213003, P.R. China

E-mail: yangchuntz@sina.com

Key words: lipopolysaccharide, cognitive dysfunction, pro-inflammatory cytokines, amyloid- $\beta$, brain-derived neurotrophic factor
$(\mathrm{P}<0.05)$. However, the administration of LPS did not elicit a statistically significant change in the expression level of BDNF in the hippocampus, compared with that in the control group $(\mathrm{P}>0.05)$. The results indicate that LPS induces cognitive dysfunction, which is associated with increased expression levels of pro-inflammatory cytokines and $A \beta$, but does not affect the expression of BDNF in the hippocampus.

\section{Introduction}

Cognitive dysfunction is a symptom characterized by dysfunction in intellectual performance and learning $(1,2)$. However, its pathogenesis has yet to be fully elucidated. Increasing evidence has shown that infection of the central nervous system is associated with the pathogenesis of cognitive dysfunction.

Lipopolysaccharide (LPS) is a cell wall component of Gram-negative bacteria and induces neuronal death, inhibits neurogenesis and impairs synaptic plasticity and memory (3-6). Previous studies have indicated that peripheral administration of LPS causes functional impairments in the brain $(7,8)$. LPS-induced peripheral infection activates the immune system, which conveys a message to the brain causing the production of inflammatory cytokines. Excessive expression of pro-inflammatory cytokines in the brain may cause behavioral deficits $(9,10)$. Regulating the inflammatory response in the brain following a peripheral infection may be important in protection against behavioral disorders (7). Moreover, an LPS-induced inflammatory response is characterized by an increased expression of pro-inflammatory cytokines, which include interleukin (IL)-1 $\beta$ and tumor necrosis factor- $\alpha$ (TNF- $\alpha$ ). Chronic activation of pro-inflammatory cytokines has been indicated to be a pivotal factor in the development of cognitive impairment (11-14). Although large studies have raised the possibility that pro-inflammatory cytokines are implicated in cognitive impairments induced by the peripheral administration of LPS, other studies have not found that cognitive deficits are improved following antibiotic treatment $(15,16)$.

In addition, a possible mechanism by which the peripheral inflammatory response may affect cognitive function is via interference with the expression of amyloid- $\beta(A \beta)$ 
and brain-derived neurotrophic factor (BDNF) $(17,18)$. The aim of the present study was to investigate the behavioral performance of rats receiving intraperitoneal injections of LPS and to determine the expression levels of $\mathrm{A} \beta, \mathrm{BDNF}$ and pro-inflammatory cytokines in the hippocampus.

\section{Materials and methods}

Animals and drugs. In total, 30 male Wistar rats weighing 180-220 g were purchased from the Shanghai Animal Center (Shanghai, China). The rats were housed five per cage with access to food and water ad libitum and were maintained on a 12-h light/dark cycle (lights on at 07:00 a.m.). Rats were randomly divided into three groups $(\mathrm{n}=10$ each) and were intraperitoneally administered saline or LPS (Sigma-Aldrich, St. Louis, MO, USA) at a dose of $250 \mu \mathrm{g} / \mathrm{kg}$ for 3 or 7 days consecutively. The experimental procedures were approved by the Institutional Animal Ethics Committee of Soochow University (Changzhou, China).

Morris water maze. Following intraperitoneal injections of LPS for 3 or 7 days, the Morris maze test was conducted to measure the cognitive function of the rats. As previously described (19), the water maze model was performed in a circular tank (diameter, $1 \mathrm{~m}$ ) filled with water. A platform was submerged below the surface of the water in the center of the target quadrant. The swimming paths of the rats were recorded by a video camera and analyzed by Videomot software (Huaibei Zhenghua Biologic Apparatus Facilities Co. Ltd., Huaibei, China). Rats were placed in the maze from four random points of the tank and were allowed to search for the platform for $60 \mathrm{sec}$. However, if this was not achieved, the rat was gently placed on the platform and left for $10 \mathrm{sec}$. The latency to the platform and the proportion of time spent in the target quadrant were recorded.

Determination of $I L-1 \beta, I L-6$ and TNF- $\alpha$ expression levels. Following the behavioral test, rats were immediately sacrificed by decapitation and the hippocampi were harvested. BDNF, IL-1 $\beta$, IL- 6 and TNF- $\alpha$ expression levels in the hippocampus were measured using a sandwich-ELISA with anti-BDNF, IL-1 $\beta$, IL-6 and TNF- $\alpha$ antibodies, according to the manufacturer's instructions (Nanjing Jiancheng Bioengineering Institute, Nanjing, China). The hippocampi were homogenized in phosphate buffer solution with $1 \mathrm{mM}$ phenylmethylsulfonyl fluoride and $1 \mathrm{mM}$ ethylene glycolO,O'-bis(2-aminoethyl)-N,N,N',N'-tetraacetic acid. Microtiter plates (96-well; flat-bottom) were coated for $24 \mathrm{~h}$ with the samples and diluted 1:2 in sample diluent. The standard curve ranged between 7.8 and $500 \mathrm{pg} / \mathrm{ml}$. Plates were washed three times with sample diluent and then monoclonal rabbit antibodies, that were diluted 1:200 in sample diluent, were added to each well. The plate was then incubated for $2 \mathrm{~h}$ at room temperature. After washing, peroxidase-conjugated anti-rabbit antibodies $(1: 2,000)$ were added to each well and the plate was incubated at room temperature for $1 \mathrm{~h}$. Following the addition of streptavidin-enzyme, substrate and stop solution, the levels of BDNF, IL-1 $\beta$, IL- 6 and TNF- $\alpha$ were determined by absorbance at $450 \mathrm{~nm}$. The standard curve demonstrated a direct relationship between optical density and BDNF, IL-1 $\beta$, IL-6 and TNF- $\alpha$ concentration. Total protein was measured by the Lowry method, using bovine serum albumin as a standard.

Determination of $A \beta$ expression levels. Total RNA was isolated from frozen muscle biopsy tissues using TRIzol reagent (Tiangen Biotech Co., Ltd., Beijing, China), according to the manufacturer's instructions. The concentration of total RNA was measured by spectrophotometry and reverse-transcribed with an RT-PCR kit (Tiangen Biotech Co., Ltd.). Quantitative PCR was performed using a SYBR Green I kit (Tiangen Biotech Co., Ltd.). Primer sequences were as follows: A $\beta$ forward, 5'-CCAGCCAATACCGAAAATGA-3' and reverse, 5'-TGATGTTTGTCAGCCCAGAA-3'; and $\beta$-actin forward, 5'-CCTGTGCTGCTCACCGAGGC-3' and reverse, 5'-GACCCCGTCTCTCCGGAGTCCATC-3'. PCR conditions were $50^{\circ} \mathrm{C}$ for $2 \mathrm{~min}, 95^{\circ} \mathrm{C}$ for $10 \mathrm{~min}$ and 40 cycles at $95^{\circ} \mathrm{C}$ for $15 \mathrm{sec}$ and $60^{\circ} \mathrm{C}$ for $60 \mathrm{sec}$.

Statistical analysis. Data are expressed as mean \pm SD. Statistical analyses were performed by one-way analysis of variance and post hoc analyses were performed using Fisher's least significant difference tests. Statistical analyses were conducted using Statistical Product for Social Sciences (SPSS), version 17.0 (SPSS, Inc., Chicago, IL, USA). P<0.05 was considered to indicate a statistically significant difference.

\section{Results}

Behavioral performance in the Morris water maze. The results of this test, which are presented in Fig. 1, indicate that the administration of LPS for 3 and 7 days significantly increased the latency to the platform and decreased the proportion of time spent in the target quadrant, compared with the control group $\left(\mathrm{F}_{(2,27)}, 11.75 ; \mathrm{P}<0.05\right.$; Fig. 1).

Expression levels of pro-inflammatory cytokines in the hippocampus. The hippocampal expression levels of IL-1 $\beta$, IL- 6 and TNF- $\alpha$ showed significant increases in the rats undergoing LPS administration for 3 and 7 consecutive days, compared with the levels in the control group. $\left(\mathrm{F}_{(2,27)}, 26.21, \mathrm{P}<0.01\right.$; Fig. 2).

Expression levels of $A \beta$ in the hippocampus. As demonstrated in Fig. 3, no significant change in the hippocampal expression level of $A \beta$ was observed following the administration of LPS for three consecutive days compared with that in the control group ( $\mathrm{P}>0.05)$. However, 7 consecutive days of LPS administration induced a significant increase in the expression level of $\mathrm{A} \beta$ compared with that in the control $\left(\mathrm{F}_{(2,27)}, 9.87 ; \mathrm{P}<0.05\right)$.

Expression levels of BDNF in the hippocampus. The results presented in Fig. 4 show that there were no significant changes in the expression levels of BDNF in the hippocampus following the administration of LPS for 3 or 7 consecutive days $\left(F_{(2,27)}\right.$, $1.43 ; \mathrm{P}>0.05)$.

\section{Discussion}

The results of the Morris water test conducted in the present study demonstrate that intraperitoneally administered LPS 
A

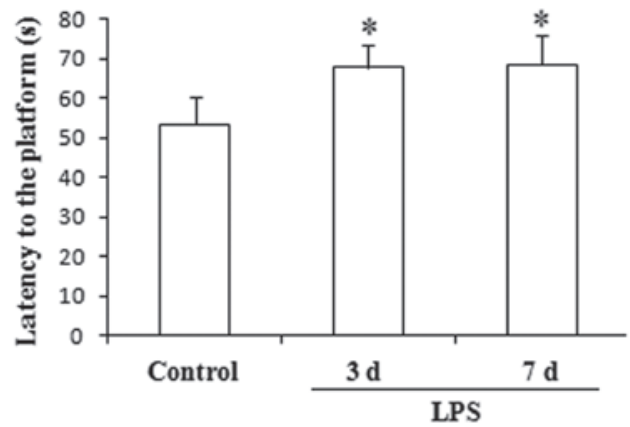

B

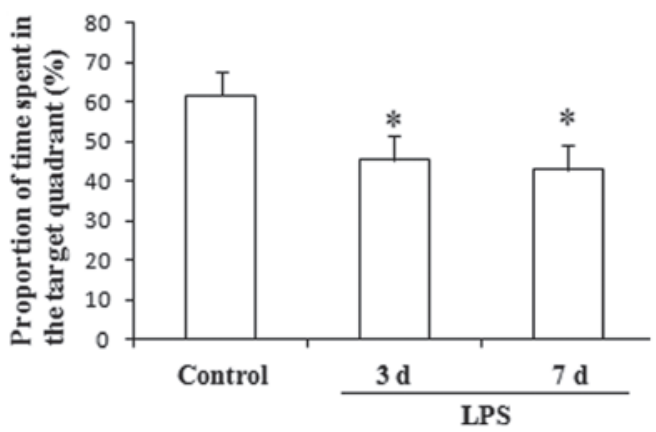

Figure 1. Behavioral performance of rats in the Morris water maze. Effect of LPS on the (A) latency to the platform and (B) proportion of time spent in the target quadrant. "P<0.05, vs. control. LPS, lipopolysaccharide.

A

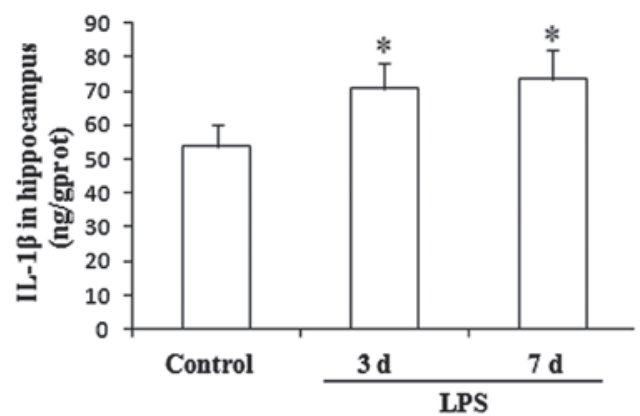

B
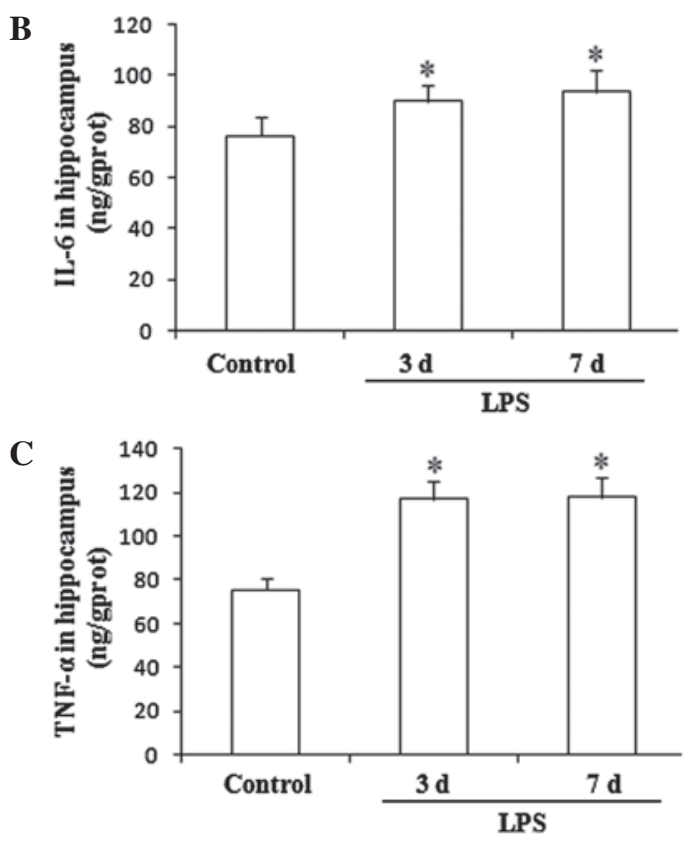

Figure 2. Effect of LPS on the expression levels of (A) IL-1 $\beta$, (B) IL-6 and (C) TNF- $\alpha$ in the rat hippocampus. "P $<0.05$, vs. control, ${ }^{\prime \prime} \mathrm{P}<0.05$, vs. LPS $3 \mathrm{~d}$. LPS, lipopolysaccharide; IL, interleukin; TNF, tumor necrosis factor.

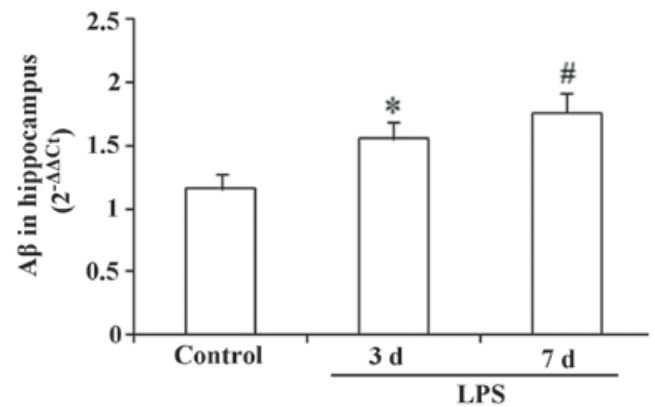

Figure 3. Effect of LPS on the expression level of A $\beta$ in the rat hippocampus. ${ }^{*} \mathrm{P}<0.05$, vs. control. LPS, lipopolysaccharide; $\mathrm{A} \beta$, amyloid- $\beta$.

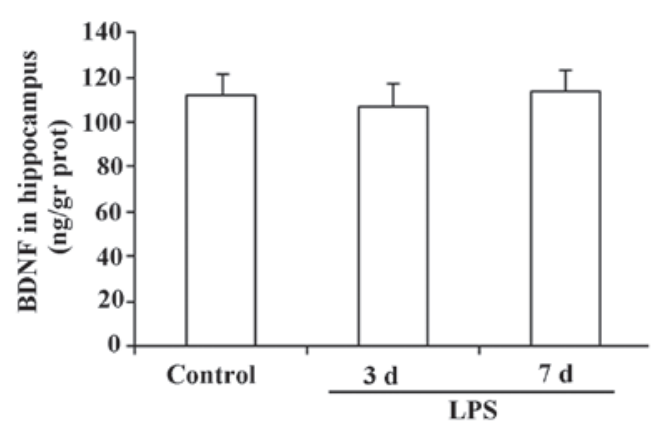

Figure 4. Effect of LPS on the expression level of BDNF in the rat hippocampus. LPS, lipopolysaccharide; BDNF, brain-derived neurotrophic factor.

elicited cognitive dysfunction in rats. Moreover, it was observed that LPS significantly increased the expression levels of pro-inflammatory cytokines and $\mathrm{A} \beta$ in the hippocampus. However, the previously expected reduction in the expression levels of BDNF was not observed.

In the Morris water maze, latency to the platform and the proportion of time spent in the target quadrant are two important testing indices for evaluating cognitive function in a rat model. In the present study, the results demonstrated that the chronic administration of LPS significantly increased the latency to the platform and decreased the proportion of time spent in the target quadrant, indicating that LPS elicited a deficit in cognitive performance.

LPS is a key component of the cell wall in Gram-negative bacteria and has the potential to cause sepsis, shock and microcirculation disturbance (20). Increasing evidence has shown that LPS may be administered to construct animal models of neurological diseases (21). Shaw et al (22) indicated that a single administration of LPS elicited cognitive impairments. In the present study, rats were intraperitoneally injected with LPS for 3 or 7 days in order to observe its effects on cognitive performance. Dantzer et al (9) hypothesized that following a single injection of LPS, particularly a short time after the administration, animals exhibit sickness behavior rather than cognitive impairment.

Pro-inflammatory cytokines are regulators of host responses to infection, immune responses, inflammation and trauma and worsen disease progression. A study by Leung et al (23) showed that increased levels of pro-inflammatory cytokines in the brain were associated with the pathogenesis of cognitive disturbance in patients with Alzheimer's disease. Moreover, 
a previous study indicated that the increased expression of pro-inflammatory cytokines facilitated the emergence of cognitive impairments (24). These observations indicate that pro-inflammatory cytokines play a pivotal role in the pathogenesis of specific diseases characterized by cognitive impairments. In the present study, the expression levels of hippocampal IL-1 $\beta$, IL- 6 and TNF- $\alpha$ were observed and showed a significant increase following the chronic administration of LPS. Therefore, the results are consistent with previous observations.

$\mathrm{A} \beta$ is a component of the amyloid plaques that are associated with Alzheimer's disease (25). A $\beta$ is a highly multifunctional peptide with significant non-pathological activity (26). In the present study, increased expression of $A \beta$ in the hippocampus was observed following the chronic administration of LPS, indicating that the increased expression of $A \beta$ may be a major factor in the pathogenesis of cognitive dysfunction. Notably, administration of LPS for 7 days elicited an increase in the expression level of A $\beta$, while 3 days of LPS administration did not. The chronic administration of LPS increased the expression levels of pro-inflammatory cytokines in the hippocampus. Therefore, it was hypothesized that high expression levels of $\mathrm{A} \beta$ may be associated with increased pro-inflammatory cytokine levels in the hippocampus. In other words, long-term infection in the central nervous system may upregulate the expression of $A \beta$. Conversely, inhibiting the inflammatory response may facilitate the downregulation of $A \beta$ (26). The results of the present study indicate that chronic LPS administration eliciting the upregulation of $A \beta$ in rat hippocampus may be associated with the observed increase in the levels of pro-inflammatory cytokines.

BDNF is a member of the neurotrophin family of growth factors, which act on certain neurons of the central and peripheral nervous system (27). Lapchak et al (28) demonstrated that the administration of LPS reduced BDNF mRNA expression levels in the rat hippocampus. Moreover, several other studies have demonstrated that proinflammatory cytokines inhibit BDNF expression in the brain $(29,30)$. Schnydrig et al (31) reported that synaptosomal BDNF expression levels in mice showed a transient reduction following the intraperitoneal administration of a single high dose of LPS, with a maximal reduction at day 3. Although previous studies have reported that BDNF plays a critical role in cognitive function, in the present study, changes in BDNF expression levels following LPS administration were not observed. Regarding the reason for this, it was considered that the LPS-elicited cognitive impairment animal model was not associated with changed BDNF expression levels. Due to this limitation, the possibility that intracranial injections of BDNF improve cognitive performance in LPS-induced cognitive impairment animal models was not investigated. Future studies are required to further investigate the correlation between LPS-induced cognitive impairment and BDNF expression.

In conclusion, LPS-induced cognitive dysfunction is likely to be associated with pro-inflammatory cytokines and $\mathrm{A} \beta$. The results of the present study indirectly indicate that early intervention against the inflammatory responses may be a strategy for attenuating the increased expression of hippocampal $A \beta$. However, the present study did not investigate drug treatments that have the potential to intervene in the expression of pro-inflammatory cytokines and ultimately reverse the emergence of cognitive dysfunction. Consequently, future large-scale studies are required to further explain the pathogenesis of cognitive dysfunction.

\section{References}

1. Adam N, Kandelman S, Mantz J, Chrétien F and Sharshar T: Sepsis-induced brain dysfunction. Expert Rev Anti Infect Ther 11: 211-221, 2013.

2. Zampieri FG, Park M, Machado FS and Azevedo LC: Sepsis-associated encephalopathy: not just delirium. Clinics (Sao Paulo) 66: 1825-1831, 2011.

3. Guan Z and Fang J: Peripheral immune activation by lipopolysaccharide decreases neurotrophins in the cortex and hippocampus in rats. Brain Behav Immun 20: 64-71, 2006.

4. Suzumura A, Takeuchi H, Zhang G, Kuno R and Mizuno T: Roles of glia-derived cytokines on neuronal degeneration and regeneration. Ann NY Acad Sci 1088: 219-229, 2006.

5. Wang Y, Cui XL, Liu YF, et al: LPS inhibits the effects of fluoxetine on depression-like behavior and hippocampal neurogenesis in rats. Prog Neuropsychopharmacol Biol Psychiatry 35: 1831-1835, 2011.

6. Deng XH, Ai WM, Lei DL, Luo XG, Yan XX and Li Z: Lipopolysaccharide induces paired immunoglobulin-like receptor B (PirB) expression, synaptic alteration, and learning-memory deficit in rats. Neuroscience 209: 161-170, 2012.

7. Richwine AF, Sparkman NL, Dilger RN, Buchanan JB and Johnson RW: Cognitive deficits in interleukin-10-deficient mice after peripheral injection of lipopolysaccharide. Brain Behav Immun 23: 794-802, 2009.

8. Sparkman NL, Buchanan JB, Heyen JR, Chen J, Beverly JL and Johnson RW: Interleukin-6 facilitates lipopolysaccharide-induced disruption in working memory and expression of other proinflammatory cytokines in hippocampal neuronal cell layers. J Neurosci 26: 10709-10716, 2006.

9. Dantzer R, O'Connor JC, Freund GG, Johnson RW and Kelley KW: From inflammation to sickness and depression: when the immune system subjugates the brain. Nat Rev Neurosci 9: 46-56, 2008.

10. Smith CJ, Emsley HC, Udeh CT, et al: Interleukin-1 receptor antagonist reverses stroke-associated peripheral immune suppression. Cytokine 58: 384-389, 2012.

11. Krzyszton CP, Sparkman NL, Grant RW, et al: Exacerbated fatigue and motor deficits in interleukin-10-deficient mice after peripheral immune stimulation. Am J Physiol Regul Integr Comp Physiol 295: R1109-R1114, 2008.

12. Johnston H, Boutin $\mathrm{H}$ and Allan SM: Assessing the contribution of inflammation in models of Alzheimer's disease. Biochem Soc Trans 39: 886-890, 2011.

13. Kaster MP, Gadotti VM, Calixto JB, Santos AR and Rodrigues AL: Depressive-like behavior induced by tumor necrosis factor- $\alpha$ in mice. Neuropharmacology 62: 419-426, 2012.

14. Mansur RB, Zugman A, Asevedo EM, da Cunha GR, Bressan RA and Brietzke E: Cytokines in schizophrenia: possible role of anti-inflammatory medications in clinical and preclinical stages. Psychiatry Clin Neurosci 66: 247-260, 2012.

15. Pizza V, Agresta A, D'Acunto CW, Festa M and Capasso A: Neuroinflamm-aging and neurodegenerative diseases: an overview. CNS Neurol Disord Drug Targets 10: 621-634, 2011.

16. Magaki S, Mueller C, Dickson C and Kirsch W: Increased production of inflammatory cytokines in mild cognitive impairment. Exp Gerontol 42: 233-240, 2007.

17. Oral E, Canpolat S, Yildirim S, Gulec M, Aliyev E and Aydin N: Cognitive functions and serum levels of brain-derived neurotrophic factor in patients with major depressive disorder. Brain Res Bull 88: 454-459, 2012

18. Zhang XY, Liang J, Chen da C, et al: Low BDNF is associated with cognitive impairment in chronic patients with schizophrenia. Psychopharmacology (Berl) 222: 277-284, 2012.

19. D'Hooge R and De Deyn PP: Applications of the Morris water maze in the study of learning and memory. Brain Res Brain Res Rev 36: 60-90, 2001

20. Solov'eva T, Davydova V, Krasikova I and Yermak I: Marine compounds with therapeutic potential in gram-negative sepsis. Mar Drugs 11: 2216-2229, 2013. 
21. Skelly DT, Hennessy E, Dansereau MA and Cunningham C: A systematic analysis of the peripheral and CNS effects of systemic LPS, IL-1 $\beta$, TNF- $\alpha$ and IL- 6 challenges in C57BL/6 mice. PLoS One 8: e69123, 2013.

22. Shaw KN, Commins S and O'Mara SM: Lipopolysaccharide causes deficits in spatial learning in the watermaze but not in BDNF expression in the rat dentate gyrus. Behav Brain Res 124: 47-54, 2001.

23. Leung R, Proitsi P, Simmons A, et al: Inflammatory proteins in plasma are associated with severity of Alzheimer's disease. PLoS One 8: e64971, 2013.

24. Reale M, Iarlori C, Gambi F, et al: Treatment with an acetylcholinesterase inhibitor in Alzheimer patients modulates the expression and production of the pro-inflammatory and anti-inflammatory cytokines. J Neuroimmunol 148: 162-171, 2004.

25. Hsu LJ, Mallory M, Xia Y, et al: Expression pattern of synucleins (non-Abeta component of Alzheimer's disease amyloid precursor protein/alpha-synuclein) during murine brain development. J Neurochem 71: 338-344, 1998.

26. Nguyen JT, Yamani A and Kiso Y: Views on amyloid hypothesis and secretase inhibitors for treating Alzheimer's disease: progress and problems. Curr Pharm Des 12: 4295-4312, 2006.
27. Yan Q, Rosenfeld RD, Matheson CR, et al: Expression of brain-derived neurotrophic factor protein in the adult rat central nervous system. Neuroscience 78: 431-448, 1997.

28. Lapchak PA, Araujo DM and Hefti F: Systemic interleukin-1 beta decreases brain-derived neurotrophic factor messenger RNA expression in the rat hippocampal formation. Neuroscience 53: 297-301, 1993.

29. Maher FO, Martin DS and Lynch MA: Increased IL-1beta in cortex of aged rats is accompanied by downregulation of ERK and PI-3 kinase. Neurobiol Aging 25: 795-806, 2004.

30. Taishi P, Churchill L, De A, Obal F Jr and Krueger JM: Cytokine mRNA induction by interleukin-1beta or tumor necrosis factor alpha in vitro and in vivo. Brain Res 1226: 89-98, 2008.

31. Schnydrig S, Korner L, Landweer S, et al: Peripheral lipopolysaccharide administration transiently affects expression of brain-derived neurotrophic factor, corticotropin and proopiomelanocortin in mouse brain. Neurosci Lett 429: 69-73, 2007. 